TITLE:

\title{
The effect of angle and moment of the hip and knee joint on iliotibial band hardness.
}

\section{$\operatorname{AUTHOR}(S)$ :}

Tateuchi, Hiroshige; Shiratori, Sakiko; Ichihashi, Noriaki

\section{CITATION:}

Tateuchi, Hiroshige ...[et al]. The effect of angle and moment of the hip and knee joint on iliotibial band hardness.. Gait \& posture 2014, 41(2): 522-528

\section{ISSUE DATE:}

2014-11-11

URL:

http://hdl.handle.net/2433/198147

\section{RIGHT:}

(c) 2014 Elsevier. Licensed under the Creative Commons Attribution-NonCommercial-No Derivatives 4.0 International http://creativecommons.org/licenses/by-nc-nd/4.0/.; NOTICE: this is the author's version of a work that was accepted for publication in Gait \& Posture. Changes resulting from the publishing process, such as peer review, editing, corrections, structural formatting, and other quality control mechanisms may not be reflected in this document.

Changes may have been made to this work since it was submitted for publication. A definitive version was subsequently published in Gait \& Posture, VOL 41, ISSUE 2, doi:10.1016/j.gaitpost.2014.12.006.; This is not the published version. Please cite only the published version.; この論文は出版社版でありません。引用の際には出版社版をご確認ご利用くだ 
Title:

The effect of angle and moment of the hip and knee joint on iliotibial band hardness

The name(s) of the author(s):

Hiroshige Tateuchi ${ }^{1), *}$, Sakiko Shiratori ${ }^{2)}$, Noriaki Ichihashi ${ }^{1)}$

The affiliation(s) of the author(s):

1) Human Health Sciences, Graduate School of Medicine, Kyoto University, Kyoto, Japan

2) Division of Physical Therapy, Rehabilitation Units, Shiga University of Medical Science Hospital, Shiga, Japan

\section{Acknowledgement:}

We would like to thank Junji Katsuhira for his assistance in the kinematic and kinetic analysis.

\section{*Address for correspondence}

Hiroshige Tateuchi, PhD.

Assistant Professor, Human Health Sciences, Graduate School of Medicine, Kyoto University, 53 Kawara-cho, Shogoin, Sakyo-ku, Kyoto 606-8507, Japan

; Tel.: +81-75-751-3964

; Fax: +81-75-751-3909

; E-mail: tateuchi.hiroshige.8x@kyoto-u.ac.jp 


\begin{abstract}
Although several studies have described kinematic deviations such as excessive hip adduction in patients with iliotibial band (ITB) syndrome, the factors contributing to increased ITB hardness remains undetermined, owing to lack of direct in vivo measurement. The purpose of this study was to clarify the factors contributing to an increase in ITB hardness by comparing the ITB hardness between the conditions in which the angle, moment, and muscle activity of the hip and knee joint are changed. Sixteen healthy individuals performed the one-leg standing under 5 conditions in which the pelvic and trunk inclination were changed in the frontal plane. The shear elastic modulus in the ITB was measured as an indicator of the ITB hardness using shear wave elastography. The three-dimensional joint angle and external joint moment in the hip and knee joints, and muscle activities of the gluteus maximus, gluteus medius, tensor fasciae latae, and vastus lateralis, which anatomically connect to the ITB, were also measured. ITB hardness was significantly increased in the posture with pelvic and trunk inclination toward the contralateral side of the standing leg compared with that in all other conditions (increase of approximately 32\% compared with that during normal one-leg standing). This posture increased both the hip adduction angle and external adduction moment at the hip and knee joint, although muscle activities were not increased. Our findings suggest that coexistence of an increased adduction moment at the hip and knee joints with an excessive hip adduction angle lead to an increase in ITB hardness.
\end{abstract}

\title{
1. Introduction
}

Iliotibial band (ITB) syndrome is an overuse injury associated with pain on the lateral aspect of the knee. The incidence of ITB syndrome in runners has been estimated to be between 5-14\% [1]. The excessive compression between the ITB and lateral femoral epicondyle has been advocated as a cause of the ITB syndrome [2]. A recent review postulated that abnormal increase in the compression forces between the ITB and lateral femoral epicondyle causes irritation and inflammation in the tissue deep to the ITB [3]. Excessive hardness of the ITB can increase the compression force exerted by the ITB on the lateral femoral epicondyle. Thus, investigation of the factors increasing ITB hardness would shed light on the ITB syndrome.

Previous studies have investigated the biomechanics of the lower extremity during running by comparing healthy individuals and patients with ITB syndrome [4-11]; however, the research findings related to the kinematic and kinetic characteristics are controversial, probably because they have been reported in retrospective studies that include causal and compensational effects. In a prospective study, Noehren et al., [5] reported greater hip adduction and knee internal rotation throughout the stance phase of running in patients with ITB syndrome. They proposed that this motion increases ITB strain causing it to compress against the lateral femoral condyle. However, a 
weak relationship between the peak strain and strain rate, and the kinematic change of the lower extremity was reported [6]. These studies suggest that kinematic change is partly responsible for increasing strain in the ITB; however, the factors contributing to an increased ITB hardness are not well understood.

More importantly, direct in vivo ITB hardness measurements have not been reported to date. Therefore, such measurements are required to determine the factors affecting ITB hardness. Shear-wave elastography (SWE) is a reliable non-invasive ultrasonographic imaging technique for evaluating soft tissue properties by measuring the propagation velocity of the shear waves in tissues, allowing the shear elastic modulus to be calculated [12-19]. SWE has been used to measure the elastic properties of the patellar and Achilles tendons, as well as the thigh and calf muscles $[12,15,18]$. Thus, a quantitative SWE could be useful in the measurement of ITB hardness.

The ITB is a lateral thickening of the fascia in the thigh, connecting the hip and knee muscles, i.e., the gluteus maximus (Gmax), gluteus medius (Gmed), tensor fasciae latae (TFL), and vastus lateralis (VL) [20-22]. Therefore, an excessive hip adduction, increase of external joint moment, and contraction of the above-described muscles could contribute to the increase in ITB hardness. The factors contributing to an increase in ITB hardness could be revealed by comparing the ITB hardness between the conditions in which the related factors are manipulated. Consequently, we aimed to clarify the factors contributing to an increase in ITB hardness using quantitative SWE. We hypothesized that ITB hardness would be increased by the increase in external load in addition to the increase in hip adduction angle. By examining the relationship between postural characteristics and ITB hardness as a basic research, we will gain insight into the potential cause of the ITB syndrome.

\section{Methods}

\subsection{Participants}

Sixteen healthy volunteers (8 men and 8 women; age, $21.9 \pm 1.0$ (mean \pm SD) years; weight, 61.5 $\pm 10.8 \mathrm{~kg}$; height, $169.3 \pm 7.8 \mathrm{~cm}$ ) participated in this study. Exclusion criteria included the presence of disease of any joint in the lower extremity/spinal joints and neurological disease. All subjects provided informed consent and the protocol was approved by the Ethics Committee of the Kyoto University Graduate School and Faculty of Medicine.

\subsection{Experimental protocol.}

Prior to data collection for the one-leg standing, data were collected for $5 \mathrm{~s}$ in the bilateral standing position that was used as a reference for calculating the joint angle. Trials under 5 one-leg standing conditions on the dominant leg (defined as the leg the participant would use to kick a ball) were then conducted as follows (Fig. 1a): normal condition, normal one-leg standing with no pelvic and trunk inclination (NO); Pdrop, $10^{\circ}$ drop of the pelvic contralateral side without trunk inclination; PTdrop, $10^{\circ}$ drop of the pelvic contralateral side with trunk inclination toward the contralateral side; Prise, $10^{\circ}$ rise of the pelvic contralateral side without trunk inclination; and PTrise, $10^{\circ}$ rise of the 
pelvic contralateral side with trunk inclination toward the ipsilateral side. The angle change was checked by an examiner using goniometer. The contralateral hand was held at the abdomen. To maintain a stable posture, the participant was allowed to touch a fixed device with an index fingertip so that it was less likely to provide mechanical support [23]. The 5 conditions were measured in random order. ITB hardness was measured by SWE after holding each stable posture for $5 \mathrm{~s}$ [12]. The kinematic/kinetic variables and electromyographic (EMG) values were recorded synchronously for $3 \mathrm{~s}$ while maintaining each posture sequentially following the SWE measurement (Fig.1b).

\subsection{Shear-wave elastography.}

ITB hardness was measured at the level of the superior border of the patella using SWE (Aixplorer, SuperSonic Imagine, Aix-en-Provence, France) while maintaining each posture. ITB was identified by palpation and B-mode image, and the anterior and posterior borders of the ITB as well as the superior border of the patella were marked. The transducer was placed lightly with a generous amount of ultrasound gel for $5 \mathrm{~s}$ by a single investigator. In the present study, the transducer was placed transversely considering the following situation. The shear elastic modulus was consistently greater in the longitudinal than the transverse measurement [24]. In our preliminary experiment, we determined the shear elastic modulus in the ITB near the upper limit by longitudinal measurement. The reliability of the values obtained near the upper limit of the measurement device was reduced [15]. Furthermore, in the skeletal muscle, only slight increases in the shear elastic modulus with increased strain were found in transverse measurement compared to that in longitudinal measurement [17]. However, in the calcaneal tendon, elastic modulus was increased in response to tendon stretching regardless of whether the measurements were made longitudinally or transversely, and the reliability of values in the stretched positions was better in the transverse than the longitudinal measurement [15]. The inconsistency of these results may be attributed to the differences in histology and hardness of the tissue. The ITB is composed of dense fibrous connective tissue, and it is regarded as a tendinous tissue [25]. Therefore, we adopted transverse measurement to determine the ITB hardness.

Based on the thickness (approximately $1.9 \mathrm{~mm}$ ) and width (approximately $5.3 \mathrm{~mm}$ ) of the ITB determined using sonography [26,27], 3 regions of interest (ROI) with diameter of $1.5 \mathrm{~mm}$ for the measurement of Young's modulus were set horizontally to cover the entire region of the ITB and their mean was calculated (Fig. 1c). The observed values of Young's modulus were divided by 3 to obtain the shear elastic modulus. All the measurements were performed twice and the mean shear elastic modulus values for 2 trials were used in the analysis. The determination of the ROI and calculation of the shear elastic modulus were performed by 1 examiner who was blind to the experimental conditions.

\subsection{Motion capture.}

The kinematic and kinetic measurements were recorded using a 7-camera Vicon motion system (Vicon Nexus; Vicon Motion Systems Ltd. Oxford, England) at a sampling rate of $100 \mathrm{~Hz}$ and a 
fourth order Butterworth low-pass filter with a $6 \mathrm{~Hz}$ cutoff, and force plates (Kistler Japan Co., Ltd. Tokyo, Japan) at a sampling rate of $1000 \mathrm{~Hz}$ and a low-pass filter (20 Hz), respectively. Reflective markers were placed by one examiner. A total of 27 markers were placed bilaterally on the acromioclavicular joint, anterior superior iliac spine, posterior superior iliac spine, superior aspect of the greater trochanter, lateral femoral condyle, medial femoral condyle, lateral malleoli, medial malleoli, heel, fifth metatarsal head, and first metatarsal head. Additionally, markers were placed on the jugular notch, xiphoid process, seventh cervical spinous process, tenth thoracic spinous process, and right scapula. The thoracic segment had 6 markers at the jugular notch, xiphoid process, seventh cervical spinous process, tenth thoracic spinous process, and bilateral acromioclavicular joints. The pelvic segment contained 4 markers at the bilateral anterior superior iliac spine and posterior superior iliac spine. The thigh segment had 3 markers at the superior aspect of the greater trochanter and at the medial and lateral femoral condyles. The shank segment had 4 markers at the medial and lateral femoral condyles and at the medial and lateral malleoli. According to a previous study [28], we calculated the three-dimensional joint angle and external joint moment of the hip and knee joints using the BodyBuilder software (Vicon Motion Systems Ltd. Oxford, England). In the analysis, segments were regarded as rigid and the joint moment was calculated using a link segment model, in which segments were connected together at nodal points. To compute the joint moment, coordinate data were added to the GRF data, in which the position of the center of mass, the weight portion, and the moment of inertia of each segment were used as parameters. The joint center of the hip was determined by first calculating a vector linking both greater trochanter markers. The joint center of the hip was then determined at a point interpolated at a distance of $18 \%$ of the vector norm from each reflective marker of the superior aspect of the greater trochanter along the vector. The joint center of the knee was determined by identifying the mid-point of a line linking the medial femoral condyle marker to the lateral femoral condyle marker [28]. The joint angles were calculated on the basis of the joint angles in a bilateral standing position. The external joint moments were normalized for body weight and height. The participants who showed less than half of the prescribed change (i.e. $<5^{\circ}$ ) in hip adduction angle in each condition with respect to the NO condition were excluded from the analysis.

\subsection{Muscle activity.}

EMG data were collected by sampling at $1000 \mathrm{~Hz}$, using the Telemyo DTS system (Noraxon USA Inc., Scottsdale, AZ). After the electrode sites were shaved and cleaned with scrubbing gel and alcohol, disposable pre-gelled Ag-AgCl electrodes (Blue sensor; Medicotest Inc., Olstykke, Denmark) were applied over the following 4 muscles of the standing side, according to the SENIAM recommendations (SENIAM website). Subjects were instructed to contract their muscles against resistance while the EMG signals for the maximal voluntary isometric contractions (MVIC) during 3 $\mathrm{s}$ were recorded for each muscle. The raw EMG signals were processed using a bandpass filter range of 20-450 Hz. The root-mean-squares (RMSs) of the raw data were determined. The mean RMS EMG amplitude was determined over the 3-s period while each posture was maintained. The mean 
RMS EMG amplitude of each muscle was normalized for each MVIC.

\subsection{Statistical analysis.}

To assess the intra-rater reliability of the shear elastic modulus measurement, the intra-class correlation coefficients (ICC [1,2]) and standard error of measurement (SEM) were calculated for a sample of 14 participants. For each parameter, a paired $t$ test with a Shaffer correction for multiple comparisons was used to determine differences among the 5 conditions. A $P$ value $<0.05$ was considered statistically significant. SPSS version 19.0 (IBM Japan Ltd.) was used for statistical analysis.

\section{Results}

Two subjects ( 1 man and 1 woman) showed a change of $<5^{\circ}$ in the hip adduction angle in Pdrop, PTdrop, Prise, and PTrise with respect to the NO; thus, those 2 subjects were excluded from the analysis.

The excellent reliability of the SWE measurement was established. The intra-rater reliability (ICC [1,2]) was 0.94 and the SEM was $1.57 \mathrm{kPa}$.

ITB hardness was significantly increased in the PTdrop compared with that in all other conditions. Conversely, the ITB hardness in the PTrise was significantly decreased compared with that in all other conditions, except Prise. However, the significant change with respect to the NO condition was not found in Pdrop and Prise (Fig. 2).

In the kinematic analysis (Fig. 3), the contralateral side of the pelvis significantly dropped in the Pdrop and PTdrop, and rose in the Prise and PTrise, as expected. Additionally, we confirmed that the thorax was significantly inclined toward the contralateral side and ipsilateral side of the standing leg in the PTdrop and PTrise, respectively.

Compared with the NO, the hip adduction angle was significantly increased in the Pdrop and PTdrop, and decreased in the Prise and PTrise. Additionally, the hip adduction angle was not significantly different between the Pdrop and PTdrop, and between the Prise and PTrise. The hip internal rotation was increased in the Pdrop and decreased in Prise and PTrise compared with that in the other conditions. The knee internal rotation angle was significantly increased in the Prise and PTrise compared with that in other conditions, and was increased in the NO compared with that in the Pdrop. However, the changes in the rotation angle of the hip and the knee joints were relatively small.

In the kinetic analysis (Fig. 4), the external hip adduction moment was significantly increased in PTdrop compared with that in all other conditions. Conversely, the hip adduction moment in the PTrise was significantly decreased compared with that in all other conditions. There was no significant difference in the hip adduction moment among the NO, Pdrop, and Prise. In the PTdrop, the hip external rotation moment was also increased slightly compared with that in all other 
conditions. Additionally, the external knee adduction moment was significantly increased in the PTdrop and decreased in the PTrise compared with that in all other conditions, denoting the same tendency as the hip adduction moment.

With regard to the EMG analysis (Fig. 5), the activity of the Gmax was increased in the Prise compared with that in all other conditions. Gmed and TFL showed the same tendency; muscle activity was increased in the Prise compared with that in all other conditions, and decreased in the Pdrop and PTdrop compared with that in the NO, Prise and PTrise. In the VL, there was no significant difference among conditions. However, EMG values for the VL in all conditions and Gmax in 4 of the 5 conditions were $<5 \%$ MVIC; thus, the activity of those muscles cannot be attributed to positive EMG signals [29].

\section{Discussion}

Our results indicate that ITB hardness was highest in the PTdrop condition. In the PTdrop, the hip joint was in an adducted position; the finding that ITB hardness increased in the PTdrop would support previous findings indicating excessive hip adduction as a major factor for the increase in ITB stress [5,7]. However, ITB hardness was increased in the PTdrop compared with that the Pdrop, despite no significant difference in the hip adduction angle between these 2 conditions. Therefore, it is unlikely that the hip adduction angle alone affects the ITB hardness. Although an increased activity of the muscles connecting to the ITB may increase the ITB hardness in conditions with a similar joint angle as PTdrop and Pdrop, no significant difference was found in any muscle between the PTdrop and Pdrop. On the other hand, the change in the external hip adduction moment and ITB hardness showed an analogous pattern, as it was most increased in the PTdrop. As mentioned above, it is probable that the hip adduction angle is one of the most important factors contributing to increasing ITB hardness, since the ITB hardness was increased in the Pdrop compared with that in the Prise, without any significant difference in the hip adduction moment. However, the ITB hardness was different between the PTdrop and Pdrop, although the hip adduction angle did not differ, indicating that the external hip adduction moment also affects the ITB hardness. As hypothesized, these findings suggest that ITB hardness was increased especially when the hip adduction alignment coexists with an excessive hip adduction moment under weight-bearing conditions. In the PTdrop, the hip external rotation moment was also increased. However, the value was considerably lower than that in the frontal plane moment; therefore, including the effect of altered hip rotation moment on ITB hardness in our study would not be adequate.

With regard to the increase in ITB hardness in the PTdrop, the external knee adduction moment was also increased in the PTdrop compared with that in the other conditions. Since ITB is located on the outside of the hip and knee joint, ITB could passively resist the external adduction moment at the knee joint as well as at the hip joint. Therefore, the posture in the PTdrop with an increased hip and knee adduction moment is thought to increase ITB hardness. Given that the passive elastic moment 
generated by the stretching of the soft tissue produces a resultant joint moment [30], the increased ITB hardness may have partly contributed to a resistance in the increased external hip and knee adduction moment in the PTdrop as a passive elastic joint moment.

Earlier studies researching the biomechanical aspects of the patients with ITB syndrome have mainly focused on the kinematic changes of the lower extremity. Although some studies have included the external moment of the hip joint as an analysis variable [5,7,9], the combined effect of the joint angle and external moment at the hip and knee has not been well studied. Our findings suggest the importance of including the assessment of external hip and knee adduction moment along with hip adduction angle when investigating the factors that cause an increase in ITB hardness. Meanwhile, recent research indicated that there was no difference in the pelvic and trunk kinematics in the frontal plane during running between the patients with ITB syndrome and the controls [9]. However, the study was conducted in individuals who reported previous diagnosis of ITB; therefore, the causal relationship between pelvic and trunk kinematics and ITB syndrome is still unclear. Since evaluation and treatment of trunk and hip functions have been recommended [31], the relationship between pelvic and trunk stabilities and ITB syndrome needs to be investigated.

The findings of this study need to be interpreted in the context of its limitations. First, we evaluated ITB hardness in static conditions. In a simulation study, the strain rate defined as the slope of the strain-time profile rather than the peak strain of the ITB was described as a factor contributing to the development of ITB syndrome [6]. However, previous studies reported that biomechanical changes in the patients with ITB during running were found throughout the stance phase or at the midsupport phase $[5,6]$. Thus, the one-leg standing posture in the current study could be used as a substitute for the posture during the stance phase. There is no reliable method to measure ITB hardness during dynamic conditions; our findings in static conditions could be used as reference data for the relationship between posture and ITB hardness. Second, although an increased ITB hardness could be presumed to lead to increased compression between the ITB and lateral femoral epicondyle, it is not clear whether ITB hardness measured by SWE is directly related to the symptom of patients with ITB syndrome. Finally, in our study, postural changes were evaluated in the frontal plane, as the kinematic deviation of the hip joint in the frontal plane has been most commonly described in previous comparative research between patients with ITB and healthy subjects [5,7-9]. However, kinematic and kinetic changes in the sagittal and transverse planes might also affect ITB hardness. Further studies will be necessary to examine the effect of biomechanical changes in planes other than the frontal plane of the hip and knee joint on ITB hardness.

In conclusion, the ITB hardness was highest in the PTdrop condition that combined an increased hip adduction angle with an external hip and knee adduction moment. However, the independent change in the hip adduction angle or hip and knee adduction moment did not necessarily result in changes in ITB hardness. Our findings suggest that ITB hardness increases when excessive hip adduction angle and adduction moment at hip and knee joint coexist. Therefore, interventions for the ITB syndrome might need to include a reduction of the excessive hip adduction moment in addition to the correction of the kinematic deviations. 


\section{Conflict of interest statement}

None of the authors have any conflicts of interest associated with this study.

\section{Acknowledgement}

We would like to thank Junji Katsuhira for his assistance in the kinematic and kinetic analysis.

\section{References}

[1] van der Worp MP, van der Horst N, de Wijer A, Backx FJ, Nijhuis-van der Sanden MW. Iliotibial band syndrome in runners: a systematic review. Sports Med 2012;42:969-92.

[2] Fairclough J, Hayashi K, Toumi H, Lyons K, Bydder G, Phillips N, Best TM, Benjamin M. Is iliotibial band syndrome really a friction syndrome? J Sci Med Sport 2007;10:74-6.

[3] Louw M, Deary C. The biomechanical variables involved in the aetiology of iliotibial band syndrome in distance runners. A systematic review of the literature. Phys Ther Sport 2014;15:64-75.

[4] Miller RH, Lowry JL, Meardon SA, Gillette JC. Lower extremity mechanics of iliotibial band syndrome during an exhaustive run. Gait Posture 2007;26:407-13.

[5] Noehren B, Davis I, Hamill J. ASB Clinical biomechanics award winner 2006: Prospective study of the biomechanical factors associated with iliotibial band syndrome. Clin Biomech 2007;22:951-6.

[6] Hamill J, Miller R, Noehren B, Davis I. A prospective study of iliotibial band strain in runners. Clin Biomech 2008;23:1018-25.

[7] Feber R, Noehren B, Hamill J, Davis I. Competitive female runners with a history of iliotibial band syndrome demonstrate atypical hip and knee kinematics. J Orthop Sports Phys Ther 2010;40:52-8.

[8] Grau S, Krauss I, Maiwald C, Axmann D, Horstmann T, Best R. Kinematic classification of iliotibial band syndrome in runners. Scand J Med Sci Sports 2011;21:184-9.

[9] Foch E, Milner CE. Frontal plane running biomechanics in female runners with previous iliotibial band syndrome. J Appl Biomech 2014;30:58-65.

[10]Foch E, Milner CE. The influence of iliotibial band syndrome history on running biomechanics examined via principle components analysis. J Biomech 2014:47:81-6.

[11] Noehren B, Schmitz A, Hempel R, Westlake C, Black W. Assessment of strength, flexibility, and running mechanics in males with iliotibial band syndrome. J Orthop Sports Phys Ther 2014;44:217-22.

[12]Kot BC, Zhang ZJ, Lee AW, Leung VY, Fu SN. Elastic modulus of muscle and tendon with shear wave ultrasound elastography: Variations with different technical setting. PLoS One 
2012;7:e44348.

[13] Maïsetti O, Hug F, Bouillard K, Nordez A. Characterization of passive elastic properties of the human medial gastrocnemius muscle belly using supersonic shear imaging. J Biomech 2012;45:978-84.

[14] Akagi R, Takahashi H. Acute effect of static stretching on hardness of the gastrocnemius muscle. Med Sci Sports Exerc 2013;45:1348-54.

[15] Aubry S, Risson JR, Kastler A, Barbier-Brion B, Siliman G, Runge M, Kastler B. Biomechanical properties of the calcaneal tendon in vivo assessed by transient shear wave elastography. Skeletal Radiol 2013;42:1143-50.

[16] Botanlioglu H, Kantarci F, Kaynak G, Unal Y, Ertan S, Aydingoz O, Erginer R, Unlu MC, Mihmanli I, Babacan M. Shear wave elastography properties of vastus lateralis and vastus medialis obliquus muscles in normal subjects and female patients with patellafemoral pain syndrome. Skeletal Radiol 2013;42:659-66.

[17] Eby SF, Song P, Chen S, Chen Q, Greenleaf JF, An KN. Validation of shear wave elastography in skeletal muscle. J Biomech 2013;46:2381-7.

[18]Hug F, Lacourpaille L, Maïsetti O, Nordez A. Slack length of gastrocnemius medialis and Achilles tendon occurs at different ankle angles. J Biomech 2013;46:2534-8.

[19] Koo TK, Guo JY, Cohen JH, Parker KJ. Relationship between shear elastic modulus and passive muscle force: An ex-vivo study. J Biomech 2013;46:2053-9.

[20] Terry GC, Hughston JC, Norwood LA. The anatomy of the iliopatellar band and iliotibial tract. Am J Sports Med 1986;14:39-45.

[21] Vieira EL, Vieira EA, da Silva RT, Berlfein PA, Abdalla RJ, Cohen M. An anatomic study of the iliotibial tract. Arthroscopy 2007;23:269-74.

[22] Becker I, Baxter GD, Woodley SJ. The vastus lateralis muscle: An anatomical investigation. Clin Anat 2010;23:575-85.

[23]Kouzaki M, Masani K. Reduced postural sway during quiet standing by light touch is due to finger tactile feedback but not mechanical support. Exp Brain Res 2008;188:153-8.

[24]Gennisson JL, Deffieux T, Macé E, Montaldo G, Fink M, Tanter M. Viscoelastic and anisotropic mechanical properties of in vivo muscle tissue assessed by supersonic shear imaging, Ultrasound Med Biol 2010;36:789-801.

[25]Fairclough J, Hayashi K, Toumi H, Lyons K, Bydder G, Phillips N, Best TM, Benjamin M. The functional anatomy of the iliotibial band during flexion and extension of the knee: implications for understanding iliotibial band syndrome. J Anat 2006;208:309-16.

[26] Goh LA, Chhem RK, Wang SC, Chee T. Iliotibial band thickness: Sonographic measurements in asymptomatic volunteers. J Clin Ultrasound 2003;31:239-44.

[27] Wang HK, Ting-Fang Shih T, Lin KH, Wang TG. Real-time morphologic changes of the iliotibial band during therapeutic stretching; an ultrasonographic study. Man Ther 2008;13:33440.

[28] Kito N, Shinkoda K, Yamasaki T, Kanemura N, Anan M, Okanishi N, Ozawa J, Moriyama H. 
Contribution of knee adduction moment impulse to pain and disability in Japanese women with medial knee osteoarthritis. Clin Biomech 2010;25:914-9.

[29] Potvin JR, O'Brien PR. Trunk muscle co-contraction increases during fatiguing, isometric, lateral bend exertions. Possible implications for spine stability. Spine 1998;23:774-80.

[30] Yoon YS, Mansour JM. The passive elastic moment at the hip. J Biomech 1982;15:905-10.

[31]Baker RL, Souza RB, Fredericson M. Iliotibial band syndrome: Soft tissue and biomechanical factors in evaluation and treatment. PM R 2011;3:550-61. 


\section{Figure captions}

Fig. 1.

(a) Experimental conditions in the one-leg standing with applied marker set. NO, normal one-leg standing condition with no pelvic and trunk inclination; Pdrop, $10^{\circ}$ drop of the pelvic contralateral side without trunk inclination; PTdrop , $10^{\circ}$ drop of the pelvic contralateral side with trunk inclination toward the contralateral side; Prise, $10^{\circ}$ rise of the pelvic contralateral side without trunk inclination; PTrise, $10^{\circ}$ rise of the pelvic contralateral side with trunk inclination toward the ipsilateral side. (b) Iliotibial band hardness was measured by shear wave elastography, and kinematic/kinetic variables and EMG values were recorded synchronously while maintaining each posture immediately following the SWE measurement. (c) A typical example of shear elastic modulus measurement at the iliotibial band (ITB). The lower image shows a B-mode ultrasound image, and the upper image shows a color-coded box superimposed on the gray scale image. The mean Young's modulus of the 3 regions of interest (Q-box: $1.5 \mathrm{~mm}$ diameter), arranged horizontally in the ITB, was calculated. The shear elastic modulus was obtained by dividing Young's modulus by 3.

Fig. 2.

The shear elastic modulus of the iliotibial band in each condition. Data are mean (SD). The horizontal line on a graph represents a statistically significant difference $(P<0.05)$.

Fig. 3.

The pelvic and thoracic angles in the frontal plane, and the three-dimensional joint angles of the hip and knee joints in each condition. Data are mean (SD). The horizontal line on a graph represents a statistically significant difference $(P<0.05)$.

Fig. 4.

The external joint moments of the hip and knee joints in each condition. Data are mean (SD). The horizontal line on a graph represents a statistically significant difference $(P<0.05)$.

Fig. 5.

The EMG amplitude in each condition. Data are mean (SD). The horizontal line on a graph represents a statistically significant difference $(P<0.05)$. 


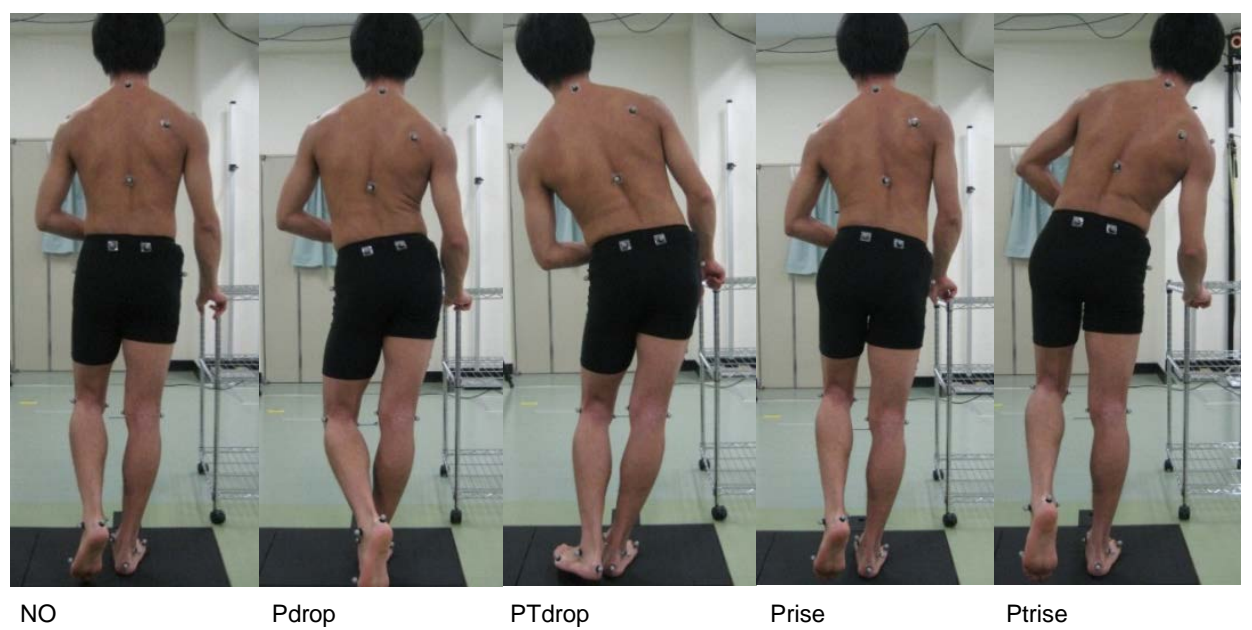

(a)


(b)

(c)

Fig 1. 


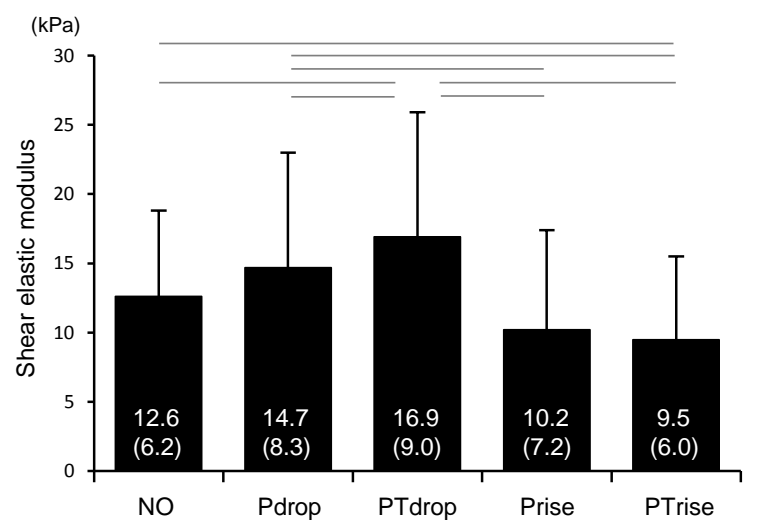

Fig 2. 

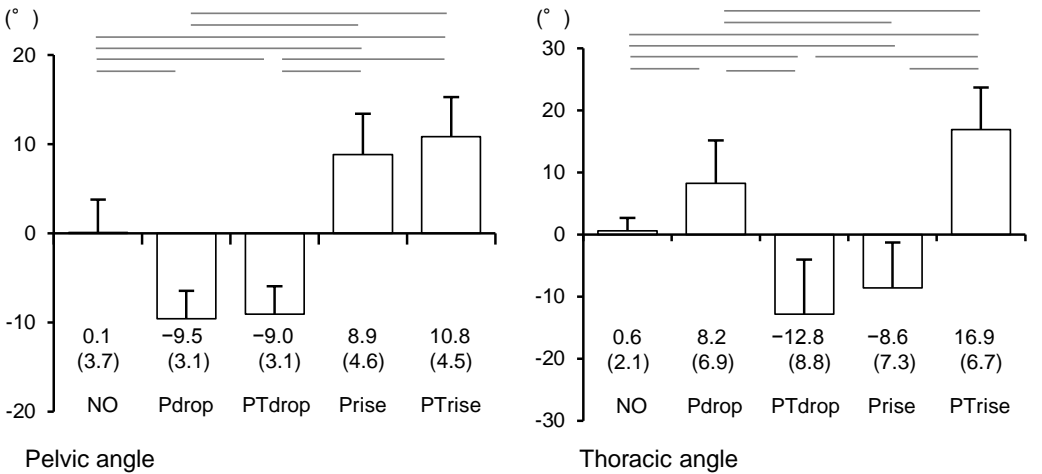

$\left({ }^{\circ}\right)$

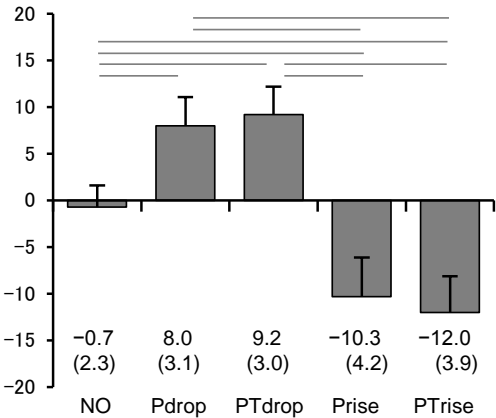

Hip adduction angle

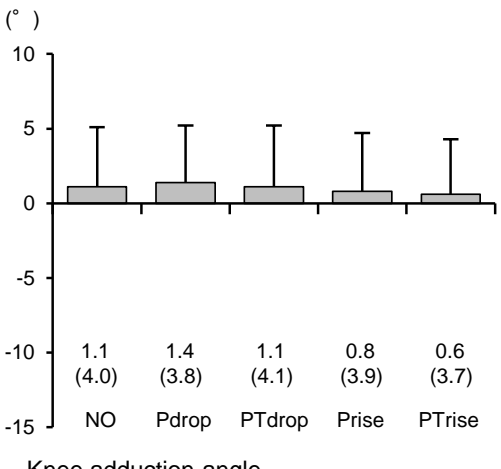

Knee adduction angle $\left({ }^{\circ}\right)$

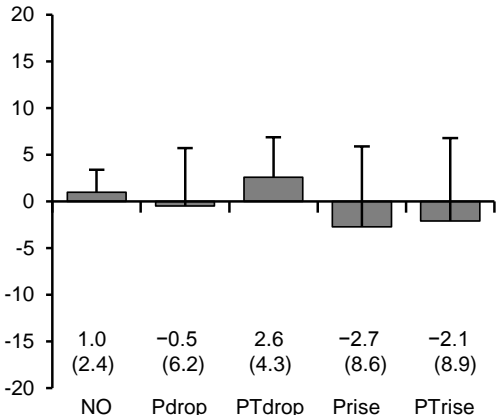

Hip extension angle

( $\left.{ }^{\circ}\right)$

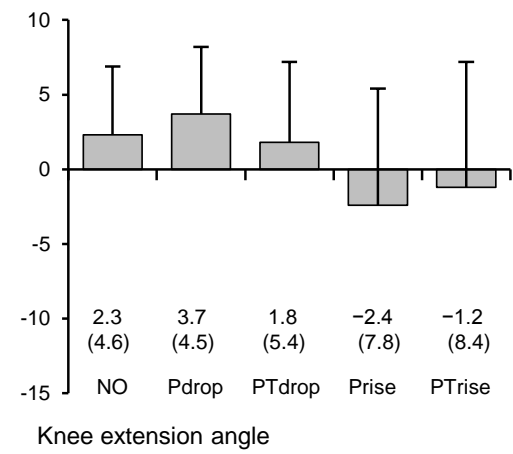

$\left({ }^{\circ}\right)$

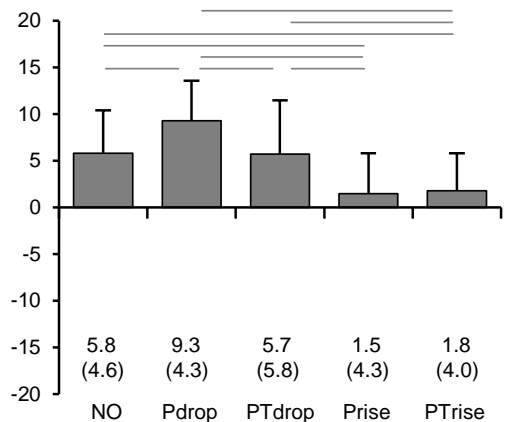

Hip internal rotation angle

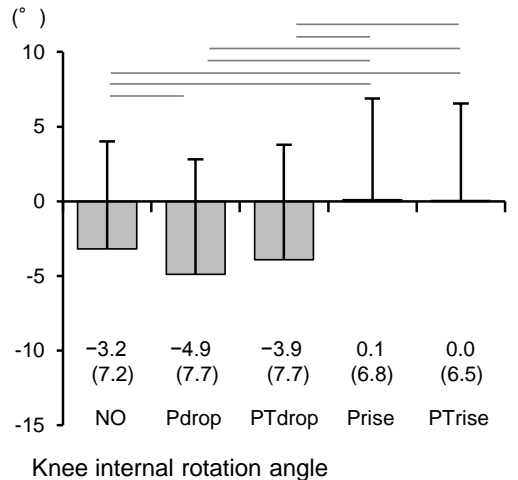

Fig 3. 


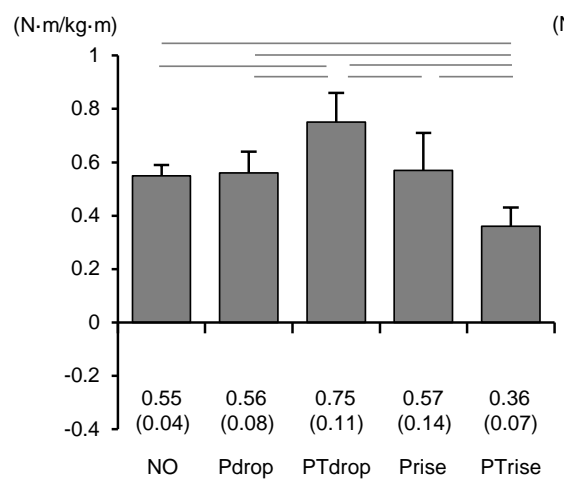

Hip adduction moment
$(\mathrm{N} \cdot \mathrm{m} / \mathrm{kg} \cdot \mathrm{m})$

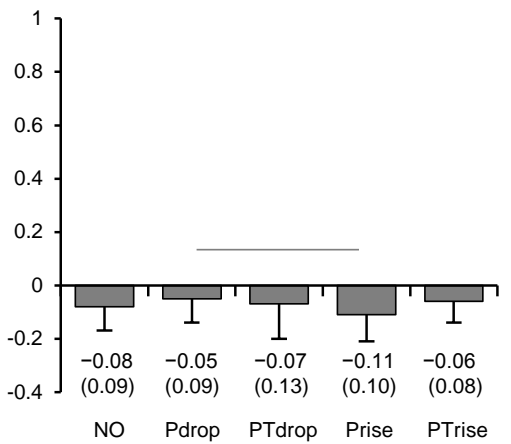

Hip extension moment
$(\mathrm{N} \cdot \mathrm{m} / \mathrm{kg} \cdot \mathrm{m})$



Hip internal rotation moment

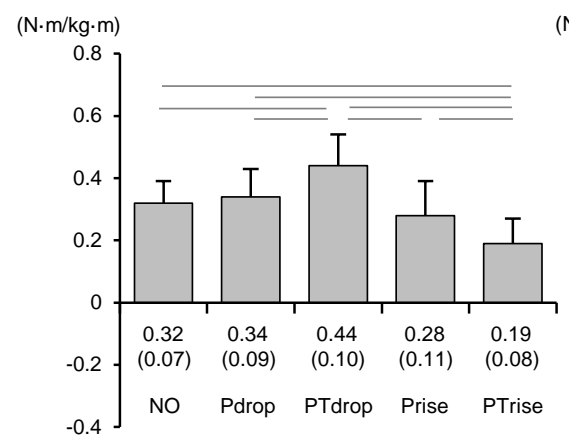

Knee adduction moment
$(\mathrm{N} \cdot \mathrm{m} / \mathrm{kg} \cdot \mathrm{m})$

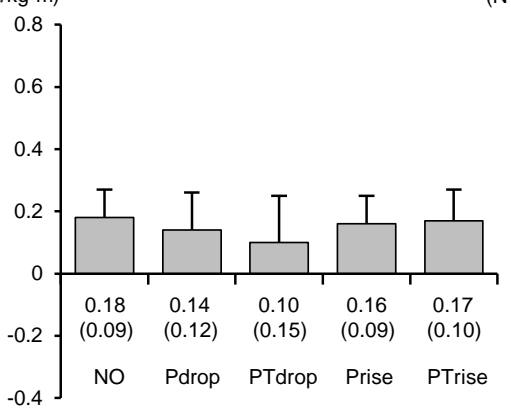

Knee extension moment
$(\mathrm{N} \cdot \mathrm{m} / \mathrm{kg} \cdot \mathrm{m})$

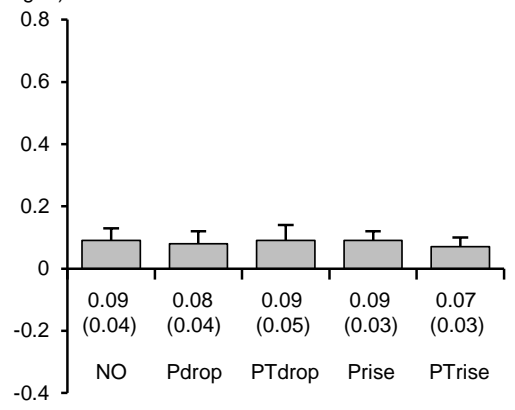

Knee internal rotation moment

Fig 4. 


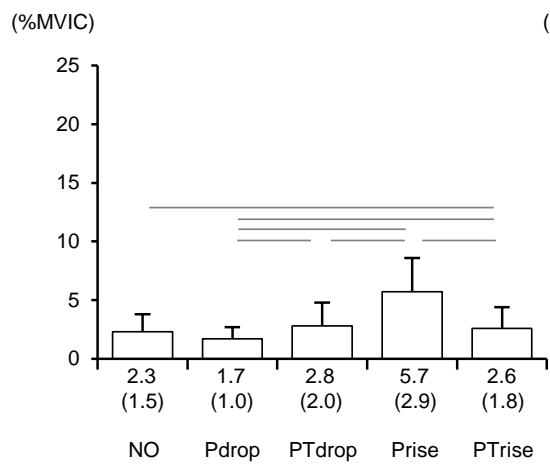

Gluteus maximus

(\%MVIC)

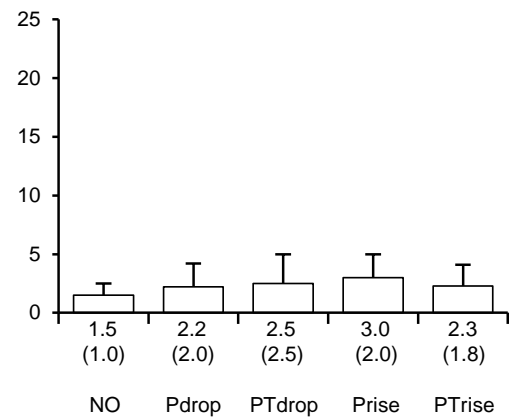

Vastus lateralis

Fig 5.

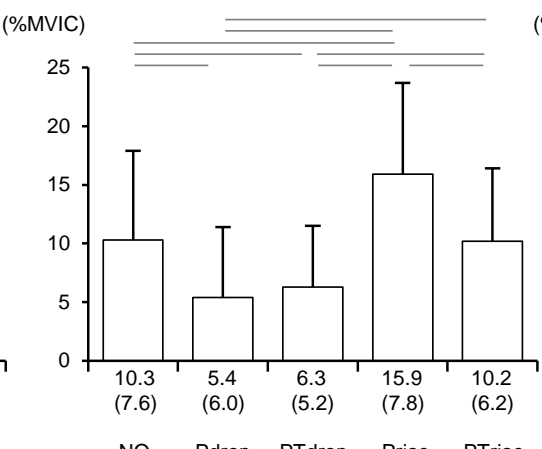

Gluteus medius
(\%MVIC)

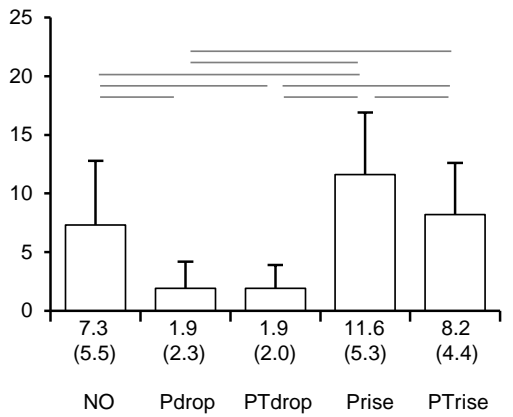

Tensor fascia latae 